\title{
Acute Hepatic Failure-An Unusual Complication of Dengue Fever in Adults
}

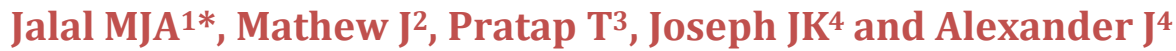 \\ ${ }^{1}$ Department of Internal Medicine and Rheumatology, VPS Lakeshore Hospital, \\ Kerala, India \\ ${ }^{2}$ Department of Family Medicine, VPS Lakeshore Hospital, Kerala, India \\ ${ }^{3}$ Department of Radiology, VPS Lakeshore Hospital, Kerala, India \\ ${ }^{4}$ Department of Internal Medicine, VPS Lakeshore Hospital, Kerala, India
}

*Corresponding author: Muhammed Jasim Abdul Jalal, Department of Internal Medicine and Rheumatology, VPS Lakeshore Hospital, Kochi, Kerala, Pin code: 682040, India, Tel: 9544020621; E-mail: poolspuff@gmail.com

\begin{abstract}
Dengue fever usually results in a mild to moderate elevation of liver enzymes. Hepatic failure is indeed a rare clinical picture in the scenario of adult dengue fever. We present a case of dengue haemorrhagic fever in an adult female, leading to acute liver failure. She was conservatively managed with adequate supportive care and discharged after 10 days. She had complete recovery and no residual symptoms were observed during follow up after one week.
\end{abstract}

Keywords: Dengue fever; liver enzymes; Haematuria; Melena; petechial rash; leucocytosis; Hepatic Failure

\section{Introduction}

Being an endemic arboviral disease, Dengue fever is a persistent public health threat in Southeast Asia. Dengue fever outbreaks have caused widespread destruction in almost all states in India. In 2010, Chrispal A et al. found that dengue fever accounted for $7 \%$ of acute undifferentiated febrile illness in adults [1]. Dengue fever spectrum ranges from mild viral fever to severe dengue hemorrhagic fever and catastrophic dengue shock syndrome. Similarly, hepatic injury spectrum in dengue fever ranges from mild - moderate elevation of liver enzymes (as seen in majority of cases) to acute hepatic failure (more common in children). We report an unusual case of an adult dengue fever leading to acute hepatic failure.

\section{Case Report}

A 48-year-old female presented with symptoms of high-grade fever, generalized myalgia and diffuse abdominal pain with non-bilious, non-projectile vomiting of 7 days duration. She also had symptoms of mucosal bleeding in the form of haematuria and melena. She did not have any past co-morbidities. Clinical examination revealed a temperature of $101^{\circ} \mathrm{F}$, pulse rate of $92 / \mathrm{min}$, blood pressure $110 / 80 \mathrm{~mm} \mathrm{Hg}$ and respiratory rate of $24 / \mathrm{min}$. She was hemodynamically stable with saturation $\left(\mathrm{SPO}_{2}\right)$ of $98 \%$ in room air. The patient was icteric and had bilateral subconjunctival haemorrhages. She had petechial spots over the soft palate and extensive petechial rash all over the trunk and limbs. Rest of the general examination was unremarkable; there was no eschar.

On systemic examination, the cardiovascular system had normal heart sounds and no murmur; examination of the chest revealed bilaterally equal air entry with basal creps; abdominal examination revealed mild hepatomegaly. She was conscious and oriented without any focal neurological deficits. 


\section{Journal of Infectious Diseases \& Travel Medicine}

Table 1 shows the patient's haematological and biochemical investigations at admission, during hospital stay and on the day of discharge. Hemogram showed leucocytosis $[15,510$ per cu $\mathrm{mm}]$ with thrombocytopenia $[25,000$ per cu $\mathrm{mm}]$. Her liver enzymes were significantly elevated [AST: 15,051 and ALT: 4122]. She also had

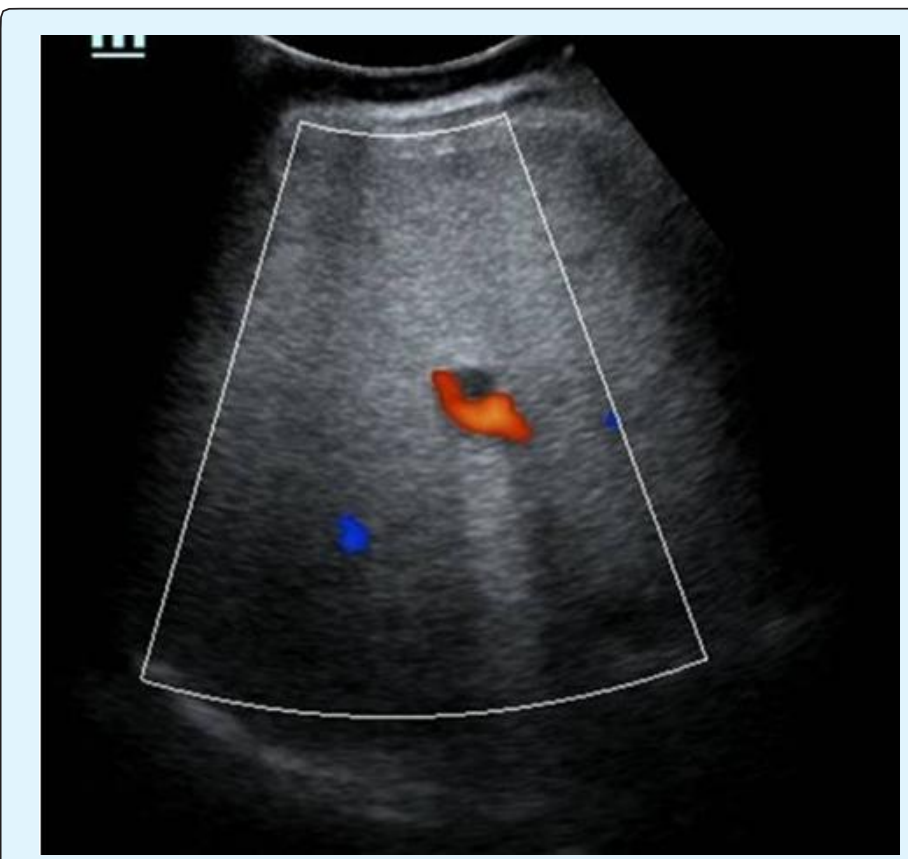

(A) deranged coagulation parameters as evidenced by a prothrombin time of 26.1 seconds and INR of 2.26. Chest radiograph revealed bilateral hazy bases. Ultrasound scan showed mild hepatosplenomegaly with Grade 2 fatty liver and Gall bladder wall thickening (Figures 1A \& 1B).

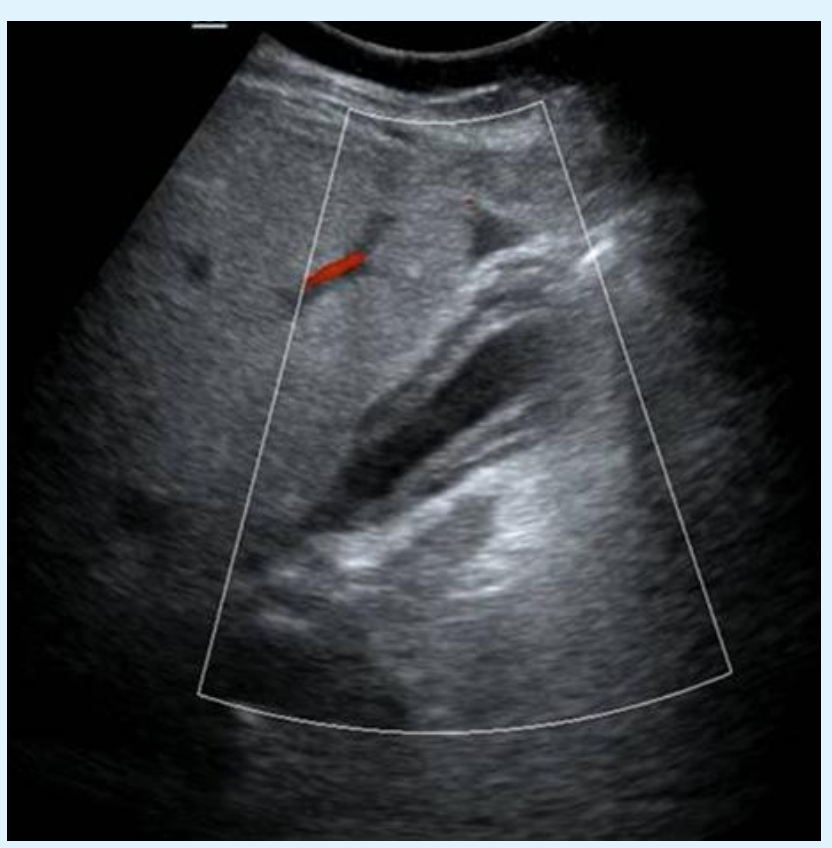

(B)

Figure 1A: USS of liver showing hepatomegaly with diffuse fatty changes.

Figure 1B: USS showing GB wall thickening with tram-track pattern (hypoechoic zone separated by two echogenic layers).

There was no ascites or pleural thickening. Plain CT showed normal lung fields with hepatomegaly (Figure 2).

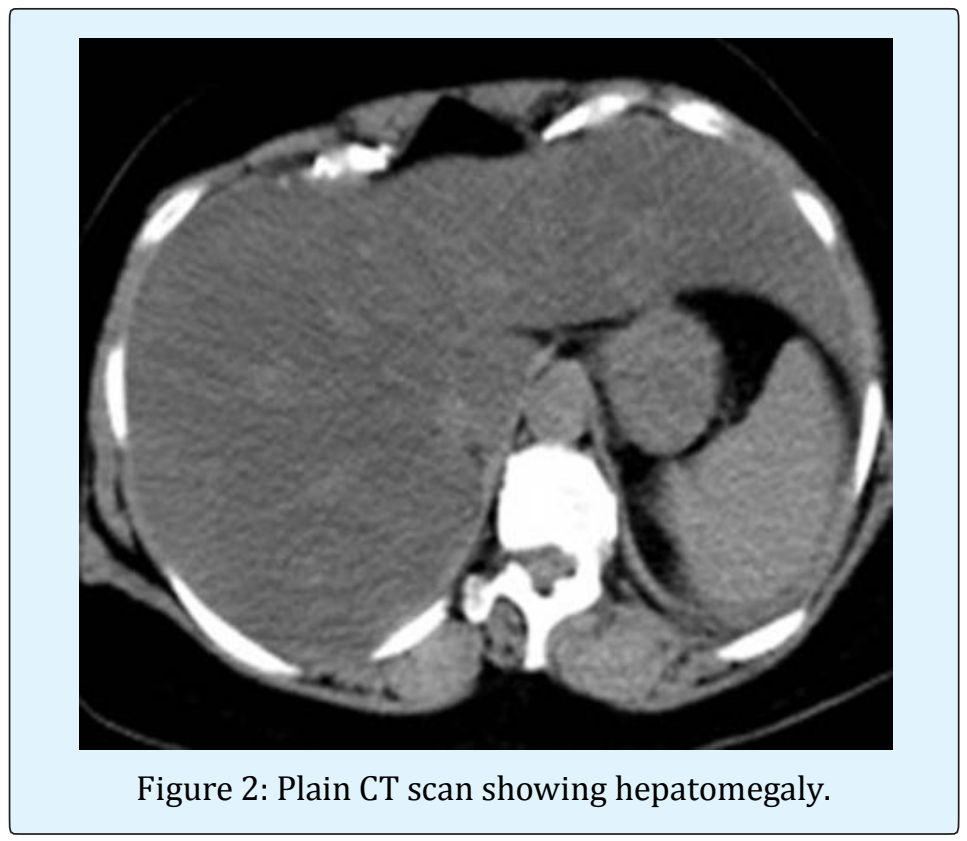

Jalal MJA, et al. Acute Hepatic Failure-An Unusual Complication of Dengue Fever in Adults. J Inf Dis Trav Med 2017, 1(1): 000102. 


\section{Journal of Infectious Diseases \& Travel Medicine}

\begin{tabular}{|c|c|c|c|c|c|c|}
\hline Day of illness & $\begin{array}{c}\text { Day of admission } \\
\text { [Day 1] }\end{array}$ & Day 2 & Day 3 & Day 4 & Day 7 & $\begin{array}{c}\text { Day of } \\
\text { Discharge }\end{array}$ \\
\hline Hb [g/dl] & 11 & 8.9 & 8.2 & 10.2 & 10.4 & 10.8 \\
\hline Total white cell count [per cu mm] & 15510 & 16270 & 14200 & 12700 & 10400 & 9550 \\
\hline Platelet count [per cu mm] & 25000 & 87000 & $1,40,000$ & $1,40,000$ & $2,44,000$ & $2,50,000$ \\
\hline Total Bilirubin [mg/dl] & 3.9 & 3.8 & 4.5 & 4.3 & 1.9 & 1.6 \\
\hline Direct Bilirubin [mg/dl] & 2.3 & 2.6 & 2.4 & 2.1 & 1 & 1.1 \\
\hline Total protein [g/dl] & 6 & 6 & 6 & 5.5 & 6 & 6 \\
\hline Albumin [g/dl] & 3.1 & 3.4 & 3.3 & 2.7 & 3.5 & 3.6 \\
\hline Aspartate aminotransferase [U/L] & 15051 & 12984 & 7803 & 4048 & 345 & 217 \\
\hline Alanine aminotransferase [U/L] & 4122 & 3260 & 2370 & 1692 & 384 & 239 \\
\hline Alkaline phosphatase [U/L] & 181 & 191 & 166 & 175 & 178 & 180 \\
\hline Creatinine [mg/dl] & 1.1 & 1.2 & ---- & ---- & 1.1 & 1.2 \\
\hline Prothrombin time [sec] & 26.1 & 24.5 & 20 & 20.6 & 19.4 & 18.2 \\
\hline International normalized ratio & 2.26 & 2.08 & 1.63 & 1.67 & 1.52 & 1.4 \\
\hline aPTT [activated partial thromboplastin time - sec] & 29 & ---- & ---- & --- & ---- & 26 \\
\hline Creatinine phosphokinase [U/L] & 140 & 144 & ---- & ---- & ---- & 148 \\
\hline Serum Sodium [mEq/L] & 130 & 135 & 136 & 139 & 136 & 136 \\
\hline Serum Potassium [mEq/L] & 4 & 4.2 & 3.8 & 3.7 & 3.4 & 3.6 \\
\hline Serum Ammonia [jg/dl] & & & $\mathbf{9 9}$ & $\mathbf{9 0}$ & $\mathbf{4 0}$ & $\mathbf{2 5}$ \\
\hline
\end{tabular}

Table 1: Laboratory profile of the patient during the course of illness.

On day 3 of hospitalization, the patient developed lethargy and a disordered sleep pattern. MRI [magnetic resonance imaging] brain and EEG [electroencephalogram] were normal. Her serum ammonia was elevated $[99 \mu \mathrm{g} / \mathrm{dl}]$. At this stage, a diagnosis of acute liver failure with hepatic encephalopathy was suspected, with aetiological possibilities of ischaemic hepatitis, drug-induced and viral-induced hepatitis. The patient's serology was positive for IgM dengue antibodies, suggestive of acute primary dengue infection. Serological tests for leptospirosis, hepatitis A (IgM), hepatitis B surface antigen [HbsAg], anti hepatitis $\mathrm{C}$ antibody and hepatitis $\mathrm{E}$ (IgM) were negative.

The patient was managed conservatively with supportive care. Since she had features of hepatic encephalopathy, she was given oral lactulose and bowel wash. She required transfusion of 6 units of platelet-rich concentrate and 3 units of FFP as she had deranged coagulation parameters. Subsequently, she remained haemodynamically stable and her blood parameters were closely monitored (Table 1); no further transfusions were found necessary.

The patient made good clinical improvement and was discharged after 10 days of hospital stay. On the day of discharge, her thrombocytopenia corrected with a platelet count of 2, 50,000 per cu mm. Her liver function improved as AST and ALT dropped to $217 \mathrm{U} / \mathrm{L}$ and $239 \mathrm{U} / \mathrm{L}$ respectively. Coagulopathy resolved as evidenced by prothrombin time of 18.2 seconds and INR 1.4. Meanwhile, the extensive petechial rashes over trunk and limbs gradually got cleared.

\section{Discussion}

The incidence of acute hepatic failure complicating dengue fever is less than 1\% [2]. In 2004, Souza LJ et al. analyzed 1585 dengue cases and did not report any complication of acute hepatic failure or hepatic encephalopathy [3]. Aspartate transaminase (AST) levels are elevated more than Alanine transaminase (ALT) levels in hepatic injury complicating dengue fever. This pattern distinguishes dengue associated liver injury from viral hepatitis, where ALT levels are higher than or equal to AST.

The pathogenesis of hepatic injury in dengue fever may be due to:

1. A direct cytopathic effect by the dengue virus within hepatocytes and Kupffer cells of infected individuals [4].

2. An immune-mediated hepatocyte injury by rapid induction of cross-reactive NS-3specific memory T-cells during a secondary infection, by the release of proinflammatory cytokines from Th2 cells [5]. 


\section{Journal of Infectious Diseases \& Travel Medicine}

Ultrasound findings of hepatosplenomegaly, GB wall thickening, pleural effusion and ascites in patients presenting with signs and symptoms of Dengue Fever are virtually diagnostic of this entity. Gall bladder wall oedema has been reported as one of the most common ultrasound findings in Dengue fever. In GB wall oedema there is preservation of mucosal echogenicity with hypoechoic appearance of muscle layer. There are 4 patterns of GB wall thickening described in Dengue fever [6]. They are A) Uniform wall thickening; B) Tram -track pattern; C) Asymmetric wall thickening; D) Honeycomb pattern. Uniform wall thickening was mostly seen in mild forms while tram- track or honeycomb pattern should alert possibility of severe forms of Dengue. Our case had tram -track pattern of wall thickening with central hypoechoic zone in between two echogenic layers indicating severe form of Dengue fever.

Supportive measures focusing on hydration and hemodynamic stability with serial monitoring of liver enzymes and coagulation parameters forms the crux of acute hepatic failure management. Sepsis and systemic inflammatory response syndrome should be anticipated by close surveillance periodically and treated promptly with initiation of adequate antibiotics. Fresh frozen plasma and platelet transfusion should be reserved for active bleeding and thrombocytopenia respectively.

Dengue fever related hepatic failure has considerably low case fatality in contrast to other aetiologies of acute hepatic failure in adults. In 2013, Tan SS and Bujang MA, described eight patients managed with standard medical therapy alone having $100 \%$ survival rates [7]. Dengue fever in children usually has a relatively higher rate of acute fulminant hepatic failure leading to $50 \%$ mortality [8]. Likelihood of spontaneous recovery in dengue related acute hepatic failure can be determined by King's College criteria or Model for End-Stage Liver Disease (MELD) score.

\section{References}

1. Chrispal A, Boorugu H, Gopinath KG (2010) Acute undifferentiated febrile illness in adult hospitalized patients: the disease spectrum and diagnostic predictors-an experience from a tertiary care hospital in South India. Trop Doct 40(4): 230-234.

2. Trung DT, Thao LTT, Hien TT (2010) Liver involvement associated with dengue infection in adults in Vietnam. Am J Trop Med Hyg 83(4): 774780 .

3. Souza LJ, Alves JG, Nogueira RMR (2004) Aminotransferase changes and acute hepatitis in patients with dengue fever: analysis of 1,585 cases. Braz J Infect Dis 8(2): 156-163.

4. Samanta J, Sharma V (2015) Dengue and its effects on liver. World J Clin Cases 3(2): 125-131.

5. Arora S, Nathaniel SD, Paul JC, Hansdak SG (2015) Acute liver failure in dengue haemorrhagic fever. BMJ Case Reports bcr 2015-209443.

6. Parmar JP, Mohan C, Vora M (2017) Patterns of Gall Bladder Wall Thickening in Dengue Fever: A Mirror of the Severity of Disease. Ultrasound Int Open 3(2): E76-E81.

7. Tan SS, Bujang MA (2013) The clinical features and outcomes of acute liver failure associated with dengue infection in adults: a case series. Braz J Infect Dis 17(2): 164-169.

8. Poovorawan Y, Hutagalung Y, Chongsrisawat V (2006) Dengue virus infection: a major cause of acute hepatic failure in Thai children. Ann Trop Paediat 26(1): 17-23. 